
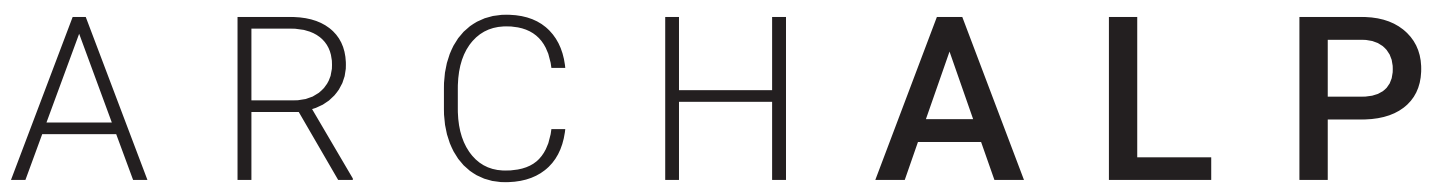

Rivista internazionale di architettura e paesaggio alpino / Revue internationale d'architecture et de paysage dans les Alpes / Internationale Zeitschrift für Alpine Architektur und Landschaft / Revija za alpsko arhitekturo in pokrajino / International journal of alpine architecture and landscape 


\title{
"Slow practicing": i progetti di Huang Yinwu nella storica città di Shaxi tra inclusione sociale e cultura materiale
}

\author{
"Slow practicing": Huang Yinwu's projects in the historic city of Shaxi \\ between social inclusion and material culture
}

\begin{abstract}
The Shaxi Rehabilitation Project is a comprehensive conservation project in a remote rural area of the Yunnan Province, China. A once important stopover in the mountain area on the ancient Tea and Horse Caravan Trail, Shaxi still has plenty of built heritage. The architect Huang Yinwu, who studied at the Southeast University of Nanjing, the Swiss Federal Institute of Technology Zürich (ETH) and at the Hong Kong University, worked as a leading project architect in Shaxi for more than 17 years, from 2003. The main topics raised by the case study deal with the local characters and topographical identity in the natural environment, the traditional craftsmanship and the intervention of modern constructions, the design, expressed by identifying and enhancing the value of Shaxi's cultural heritage and the conservation of cultural legacies into the framework of social and economic development. Shaxi has a strong construction tradition, but it is also very fragile. Unlike the urban area, the traditional rural construction type is a system of experience gradually broadened after ongoing trials and mistakes. This is the reason why it is challenging to have new creations and developments; the same goes for new materials and technologies. For architects like Huang Yinwu, it is necessary to transform the empirical system into a theoretical one, and then provide a reliable construction model that is suitable for local conditions and technologies. In another sense, the key is working with and training local artisans so that they may understand new materials and structures and develop their skills.
\end{abstract}

\section{Edoardo Bruno}

Architect and assistant professor at Politecnico di Torino. He completed a MArch (Master of Architecture) and a PhD from the same university, with a dissertation on the urban expansion in southern Guangzhou. Since 2015, he has been responsible for the activities of the South China - Torino Collaboration Lab. As part of the China Room research group, he was a project architect for the transformation of the Oxygen Factory within the Big Air Venue for the XXIV Olympic Winter Games, Shougang - Beijing 2022 and Executive Curator for the exhibition Eyes of the City during the 2019 edition of the UABB Biennale in Shenzhen.

\section{HaoHao Xu}

Associate Professor at the School of Architecture at the South China University of Technology, founder of the Faan Nguk Kei organization for Cantonese built heritage conservation, senior architect at Urban Elephant Architects, $\mathrm{PhD}$ in architectural history and theory, $\mathrm{PhD}$ in economics. Haohao Xu curated the global exhibition tour of YESTERDAY / TOMORROW: 50 Years of Urban Conservation and Innovation in Italy and has written numerous reviews on modern and contemporary architects.

\section{Keywords}

China, Rehabilitation, Shaxi, Yunnan, heritage. 


\section{Né contro né mainstream}

Il caso del recupero dell'antica città cinese di Shaxi, ad opera dell'architetto Huang Yinwu e della sua squadra di progettisti, rientra nel maggiore interesse che la Cina contemporanea sta concentrando verso le proprie aree rurali. Quella che Scott Rozelle e Natalie Hell (2020) chiamano la Invisible China, non ha ricevuto, a seguito delle riforme del 1978, le stesse attenzioni delle aree costiere, volte a interpretare il ruolo di volano economico per competere sulla scala globale.

Contestualmente alle riforme del 2006 Building a New Socialist Countryside (Ahlers, 2014; Ahlers, Schubert, 2009) e del 2012 Beautiful Countryside e Beautiful China, gli architetti cinesi hanno cominciato ad occuparsi in maniera sistematica del recupero delle aree rurali interne. Da piccoli casi isolati si è arrivati alla costruzione di vere e proprie narrazioni attorno a figure chiave come $\mathrm{Ru}$ ral Urban Framework, AZL Architects, Trace Architecture Office, DnA Architects, Atelier Deshau e Philip Yuan.

I loro progetti hanno contribuito a veicolare, se così si può definire, l'etichetta di "architettura vernacolare cinese", dove, a cavallo del restauro e il progetto ex novo, si sono consolidati immaginari, tipologie ricorrenti, scale di intervento confrontabili, materiali utilizzati. $\mathrm{Al}$ contrario della circolarità mediatica di alcuni casi studio ormai conclamati, i progetti di Huang Yinwu non sono facilmente reperibili al di là dei confini cinesi. Quello che trapela è piuttosto il "caso Shaxi" (Wong, 2016), riportato come pratica comunitaria d'eccellenza.

Come emerge dalle ricerche di Rural Urban Framework, gli spazi dell'abitare contadino cinese si

In apertura

Dettaglio di una scala a chiocciola realizzata nella torre d'avvistamento nei pressi della libreria Xiangfeng.

Fig. 1

II progetto di City

God Temple ancora in costruzione. che fa di sé l'abitante delle aree rurali cinesi contemporanee, il quale rimarca il rapporto simbiotico tra terra, lavoro e famiglia (Van der Ploeg, 2019). La relazione si estende a una miriade di connessioni all'interno e in prossimità della comunità stessa, allargando il concetto di famiglia alla consapevolezza di appartenere a un gruppo sociale coeso, con implicazioni sullo spazio costruito.

Tra pratiche comunitarie e nuove ambizioni, il caso studio di Shaxi ci aiuta a comprendere l'eterogeneità delle implicazioni dei programmi governativi di rivitalizzazione rurale attualmente attivi in Cina. La pratica di Huang Yinwu affianca al recupero dei luoghi la conoscenza formatasi collaborando a stretto contatto con la comunità locale, rendendo l'intervento diffuso, multiscalare e, a volte, invisibile. Un monito lanciato anche da molti altri architetti cinesi, preoccupati che gli interventi di riqualificazione possano generare effetti di gentrificazione proprio a partire dalle opportunità turistiche causate dalle loro stesse realizzazioni (Yang et al., 2018).

\section{Shaxi Rehabilitation Project (SRP)}

L'antica città di Shaxi si trova tra le montagne di Hengduan nel nord-ovest della provincia dello Yunnan. Posta ad un'altitudine di 2100 metri, Shaxi era un importante avamposto lungo la "Tea Horse Road". Il suo isolamento ha favorito, mentre altrove avanzava la modernizzazione del paese, la sopravvivenza della cultura materiale locale, anche se minata da un progressivo deterioramento.

Nel 2000 alcuni esperti dell'ETH di Zurigo vengono invitati dal governo della contea di Jianchuan a elaborare un progetto per lo sviluppo socioeconomico della valle. Denominato Shaxi Rehabilitation Project (SRP), tale progetto è stato promosso e finanziato attraverso il coinvolgimento di istituzioni e fondazioni svizzere e cinesi (Renfer, 2017). Nel 2001 la Shaxi Sifang Street, ultima strada mercatale ancora presente lungo la "Tea Horse Road", viene inclusa nella lista dei 100 siti di interesse del Patrimonio Mondiale del World Monuments Fund (WMF) e l'anno seguente viene lanciato ufficialmente il suo progetto di rivitalizzazione. Gli obiet- 
tivi del SRP ambiscono a recuperare la consistenza degli elementi del patrimonio costruito così come di quello immateriale.

Il percorso di studi di Huang Yinwu si intreccia con lo sviluppo del progetto SRP. Come molti architetti della sua generazione si forma in Cina presso un'università prestigiosa, nel suo caso la Southeast University di Nanchino, per virare successivamente su una destinazione estera di eccellenza. Nel 2002 appena terminata la propria formazione presso l'ETH, si trasferisce a Shaxi per iniziare ad occuparsi della rigenerazione locale come supervisore, iniziando così un percorso che lo porterà a ottenere l'incarico di Direttore del Shaxi Center for Rural Cooperation.

\section{Recupero e contraddizione}

Uno dei primi punti su cui si concentra il lavoro di Huang Yinwu, è la contrapposizione tra l'importazione di metodi di costruzione moderni e i saperi tradizionali tramandati da generazioni. La sua ricerca non è basata sul conflitto della prima sulla seconda, quanto piuttosto sul rilevare come la comunità locale non disponga delle competenze, o della familiarità, necessarie per maneggiare consapevolmente nuovi materiali imposti dal mercato. Un motivo che l'ha spinto a promuovere programmi di formazione per le maestranze locali.

Queste tematiche sono riprese nel progetto diffuso per il Shaxi Low Carbon Community Center del 2012. Esso ha incluso la realizzazione di una serie di interventi atti a sperimentare forme di bioedilizia nel contesto locale. L'obiettivo aspirava a verificare come materiali disponibili localmente e nuove tecnologie potessero essere incorporate nelle abitudini dei residenti, così da promuovere attorno al tema una diffusa consapevolezza. Il progetto ha riguardato la realizzazione di una serie di interventi di riqualificazione su edifici esistenti e spazi pubblici, volti a sostenere lo sviluppo ecocompatibile della valle.

Il punto nodale dell'atteggiamento di Huang Yinwu rispetto al recupero è la consapevolezza che l'intervento architettonico è prima di tutto un atto di partecipazione sociale. Il concetto di sostenibilità si liquefa così nelle reti sociali e il progettista deve tenerne conto. Se un abitante di Shaxi deve costruire una nuova abitazione, tutte le parti in legno devono prima essere lavorate dal falegname e l'assemblaggio del telaio richiede l'intervento di molte persone. Se la sua popolarità all'inter-

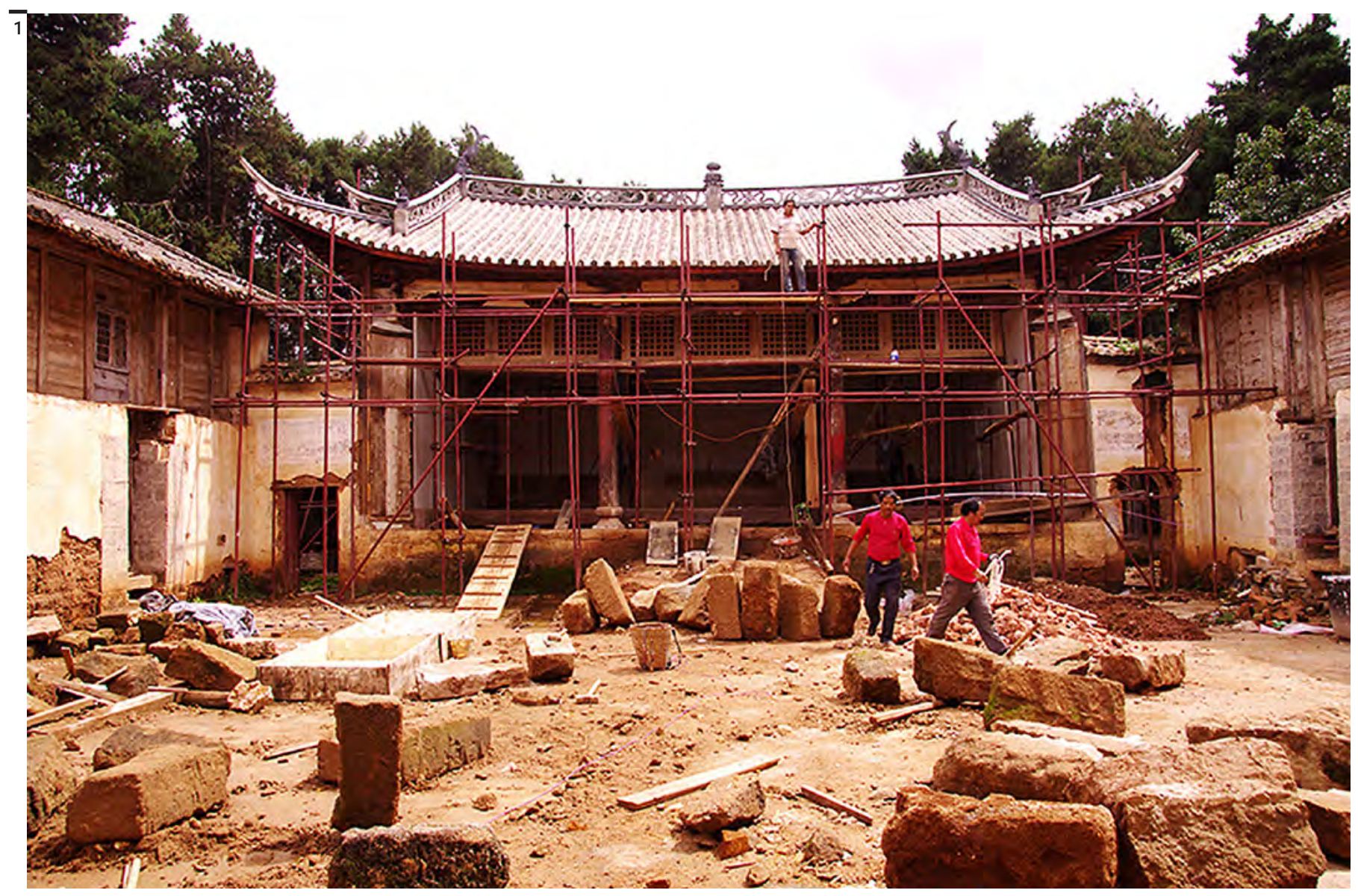


no del villaggio non è sufficiente allora quella casa non viene costruita.

L'etica ambientale non si è concentrata quindi sul mero efficientamento dell'involucro, ma su come la comunità potesse dotarsi di nuovi spazi produttivi e di condivisione sociale in una logica sostenibile, in modo tale da ridefinire l'etichetta stessa di "low-carbon" (Liu et al., 2014).

Il processo di rivitalizzazione di Shaxi non è un sistema chiuso e la marginalità iniziale nel tempo si è trasformata in una potenzialità turistica, la quale ha permesso alla comunità locale di incontrare nuovi utilizzatori provenienti dall'esterno. Come prima reazione, gli abitanti hanno cominciato ad affittare gli spazi rigenerati per trasferirsi fuori dal contesto rurale. La transizione da spazio dell'abitare a vuoto commerciale, comune in altri recenti casi studio cinesi, ha spinto il gruppo di lavoro di Huang Yinwu a trovare il mezzo attraverso cui la comunità fosse impegnata in prima linea nello sviluppo dell'economia locale. Il progetto, ancora in via di sviluppo, di City God Temple nel nuovo centro comunitario di Shaxi, ha unito l'attiva spi-

Fig. 2

Vista esterna dell'intervento della libreria Xiagfeng. governo locale ha recentemente concesso che l'edificio tornasse ad essere utilizzato a tutti gli effetti dalla comunità per raggiungere l'obiettivo del SRP di promuovere la cultura locale come volano di sviluppo economico.

Huang Yinnwu ha invitato gli artigiani locali a ricostruire il muro principale del tempio attraverso l'utilizzo della tecnica in terra cruda, e ha ricercato carpentieri provenienti dalla contea di Jianchuan per realizzare tutti gli apparati decorativi compromessi dalla storia recente: un destino comune per moltissimi centri di culto confiscati durante la Rivoluzione Culturale, e in maniera particolare lungo l'antica "Tea Horse Road" (Sigley, 2010).

La rivitalizzazione di Shaxi è passata anche attraverso la costruzione della libreria di Xiangfeng. Anche in questo caso, l'edificio da cui è stata ricavata fungeva da ex granaio. La libreria è all'interno della rete Libraire Avant-Guarde, società di Nanchino, la quale ha fatto dell'impegno di realizzare luoghi di lettura e centri culturali nelle aree più remote del paese la propria bandiera (Bruno, Tondo, 2020). La realizzazione si è basata sul recupero delle murature in terra cruda, mentre le strutture lignee interne sono rimaste pressoché intatte. Il tratto distintivo di Libraire Avant-Guarde è ben riconoscibile: investi-

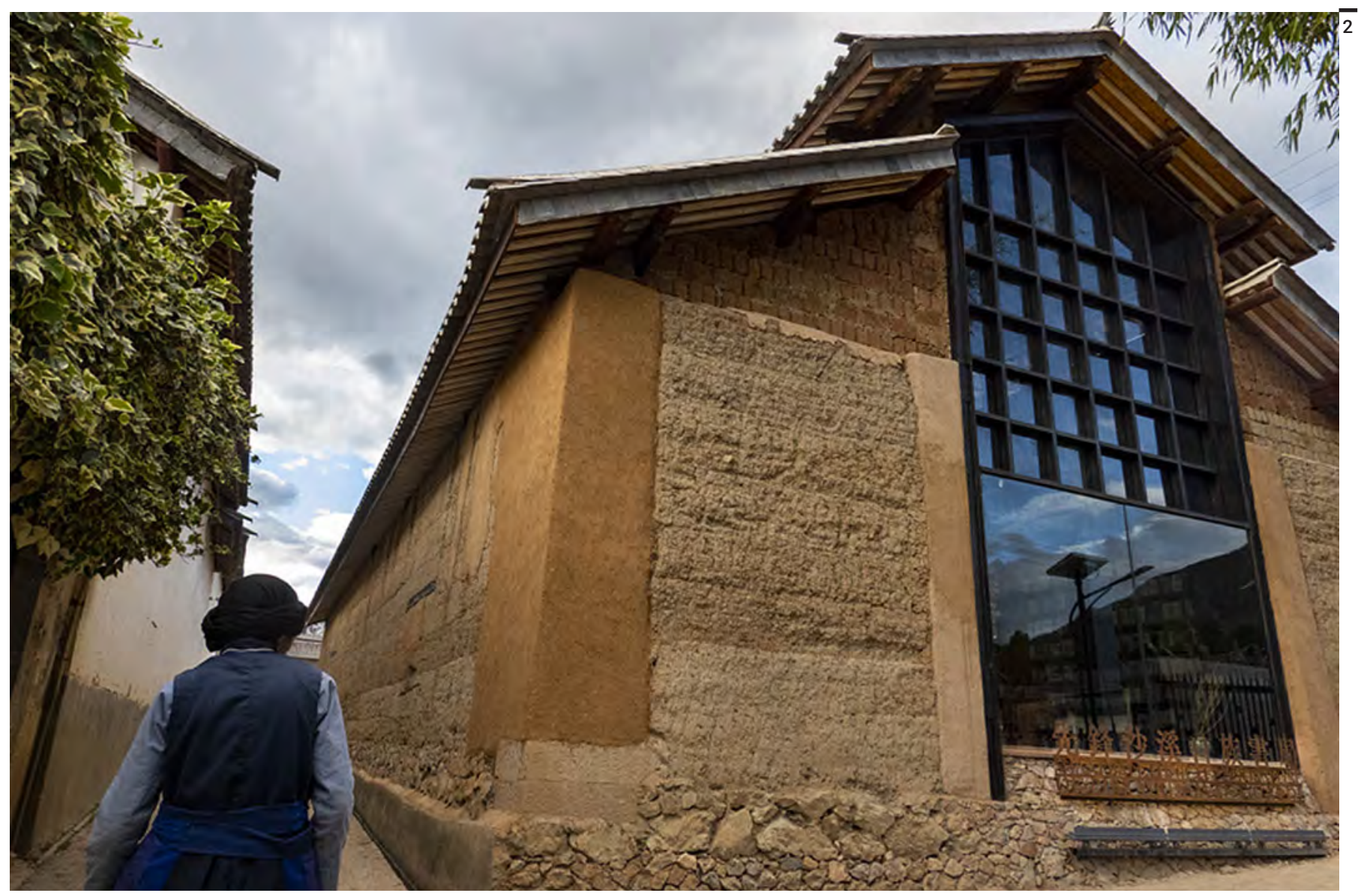


re in località fuori dai percorsi turistici più blasonati per sostenere lo sviluppo locale, selezionare edifici in disuso, fare una scelta attenta dei materiali locali al cui fianco sono disposti volumi in tutte le direzioni possibili.

\section{Luoghi, tecniche, persone}

Il progetto di recupero condotto dal team di Huang Yinwu si è basato, quindi, su un processo entro il quale le pratiche di conservazione si sono dovute intrecciare con le tecniche artigianali locali perseguendo una sinergia di saperi.

Il processo porta ad innovazioni puntuali, le quali devono però confrontarsi con la legge cinese in materia di conservazione dei beni culturali. Il principio della ricostruzione integrale del manufatto, da riparare senza alterarne la forma esistente, ha suggerito al team di operare attraverso lo stratagemma dell'inserto, piuttosto che trasformare l'impianto architettonico nella sua totalità. In questo modo il programma SRP, si è costituito su una costellazio-

Figg. 3-4 ne di mini-interventi dove coesistono tecniche, reVista interna della libreria Xiangfeng, parte del network

Libraire AvantGuarde di Nanchino. te tutte le istanze, ma come esperimento contingente e iterativo.

Nella riparazione di un gruppo di case lungo Sifang Street, le facciate in legno compromesse strutturalmente vengono consolidate con una tecnica sviluppata in Cina da secoli. I telai di copertura vengono separati rispetto alle facciate e nuovi elementi si contrappongono alla progressiva instabilità.

Il recupero del Padiglione Kui, teatro nel cuore di Shaxi, tenta di coniugare un rinnovato utilizzo degli interni con la sua memoria spaziale. Il progetto ha mantenuto l'impianto esterno, mentre i nuovi livelli hanno modificato l'esperienza di un secondo piano precedentemente dotato di altezze ridottissime. Il team di progetto ha disposto alla quota originale strisce di tessuto di colore scuro per lasciare ai visitatori la possibilità di percepire, senza urtare il soffitto, la spazialità anteriore la modifica del manufatto. L'intervento minimo vuole far riflettere sulle contraddizioni stesse del restauro contemporaneo cinese, che tende alla totale preservazione senza voler venir meno alle potenzialità offerte dai nuovi utilizzi.

Anche semplici dettagli distributivi diventano l'occasione per combinare nuovi usi e tecniche locali.

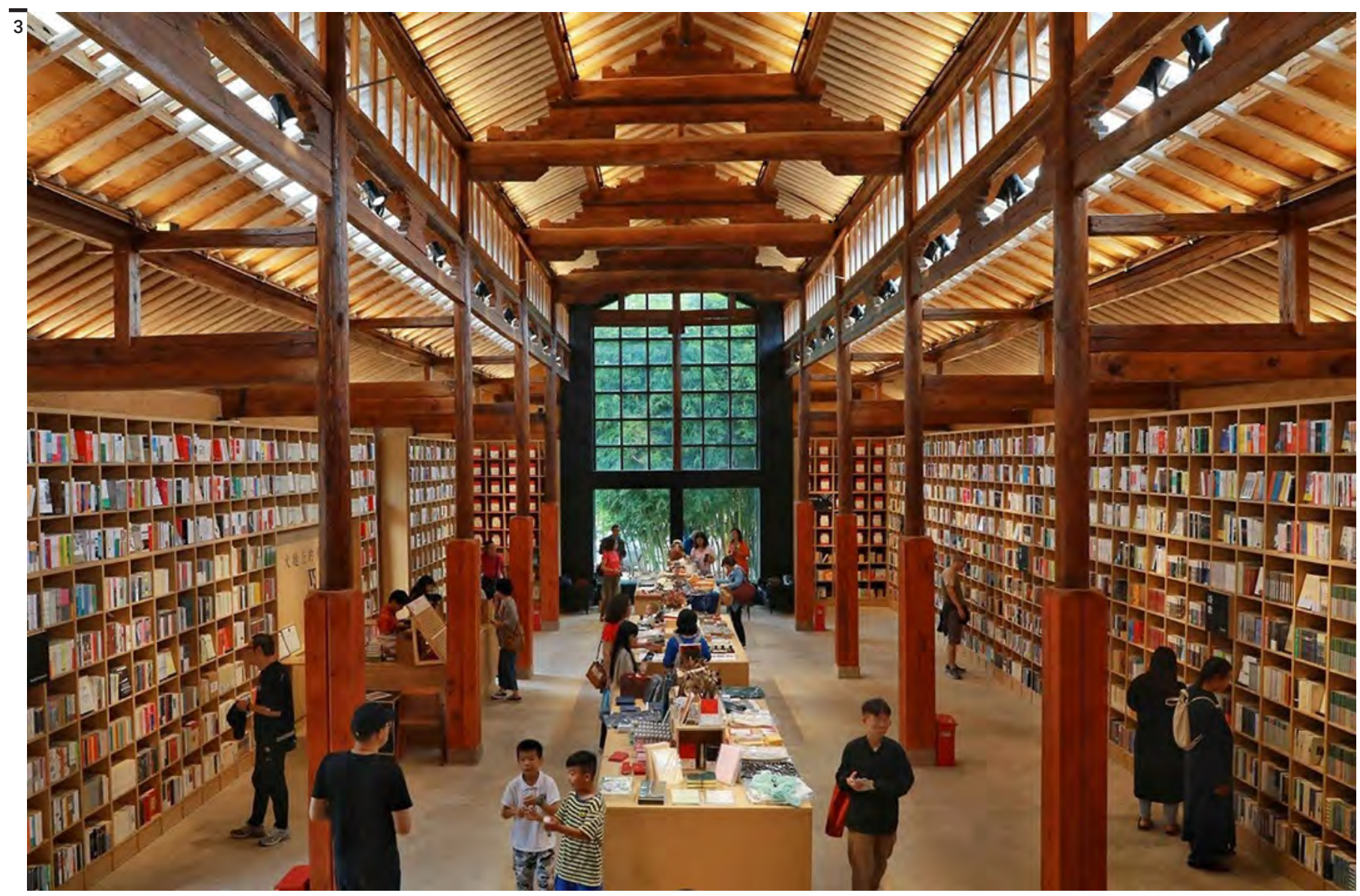




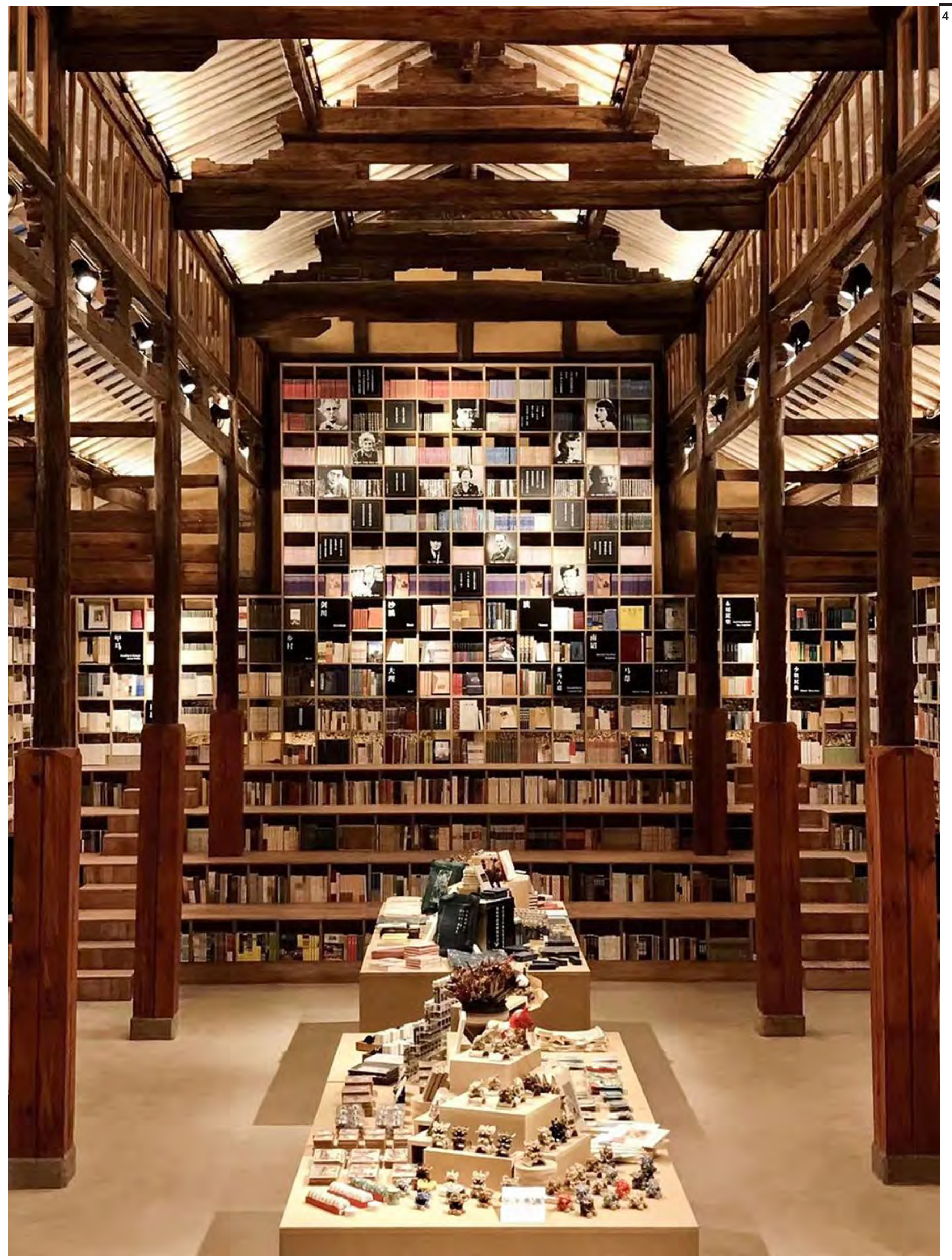


La porta di Nanzhai, un tempo fortezza di Shaxi, viene convertita in luogo di visita. Come nel caso del padiglione Kui, anche in questo caso l'accesso al secondo livello veniva utilizzato solo di rado attraverso scale molto verticali e non sempre fissate alla struttura portante. Per non alterare il manufatto, viene costruita dagli artigiani locali una scala a gradini sfalsati che, inserita nello stretto vano di risalita, permette ai visitatori di proseguire il percorso ai piani superiori. Lo spazio distributivo rimane inalterato, mentre è l'innesto ad essere visibile e realizzato per soddisfare le nuove necessità.

\section{La pratica lenta di Shaxi}

Nonostante la semplicità degli esempi sopra riportati, in realtà essi condensano tutti i più importanti principi dell'impegno profuso a Shaxi. Il recupero inteso come conoscenza del contesto sociospaziale di riferimento, le tecniche come saperi sempre in evoluzione e la preservazione del manufatto originale dove l'autorappresentazione non va oltre l'innesto ragionato, tratteggiano le modalità attorno alle quali Huang Yinwu ha posto la cultura materiale in stretta connessione al destino degli abitanti di Shaxi, prima ancora di celebrare l'architettura per sé stessa.
L'operazione portata avanti con dedizione e sostenuta dal piano SRP, può essere definita all'interno delle rapide trasformazioni architettoniche della Cina rurale come una pratica lenta. In questo modo, gli interventi su una facciata, un tempio, una scala definiscono sistemi che, a scale diverse, si mescolano alla ritualità del luogo, rinsaldandovi attorno soggetti interni ed esterni.

Il padiglione cinese dell'ultima edizione della Biennale di Venezia invita i visitatori a riflettere sul concetto della casa a corte tradizionale. Intitolato "Yuan-er, a Courtyard-ology: From the Mega to the Micro", esso pone l'accento sul ruolo di collante sociale svolto da quegli spazi capaci di intersecare la dimensione collettiva con quella domestica: a valle di decenni di sfrenata urbanizzazione, gli architetti cinesi si interrogano su quale sia l'identità degli spazi ereditati, prodotti così in fretta, o se non siano semplicemente generici.

Sulle stesse orme la lezione di Huang Yinwu è quella di associare nel recupero dei manufatti di Shaxi, una continuità tra gli spazi rinnovati e il programma della socialità che in esso continua a evolversi e modificare l'ambiente costruito. In questo senso i suoi interventi non sono pienamente formalizzati, rimangono aperti all'ibridazione e, soprattutto, allo scorrere del tempo.

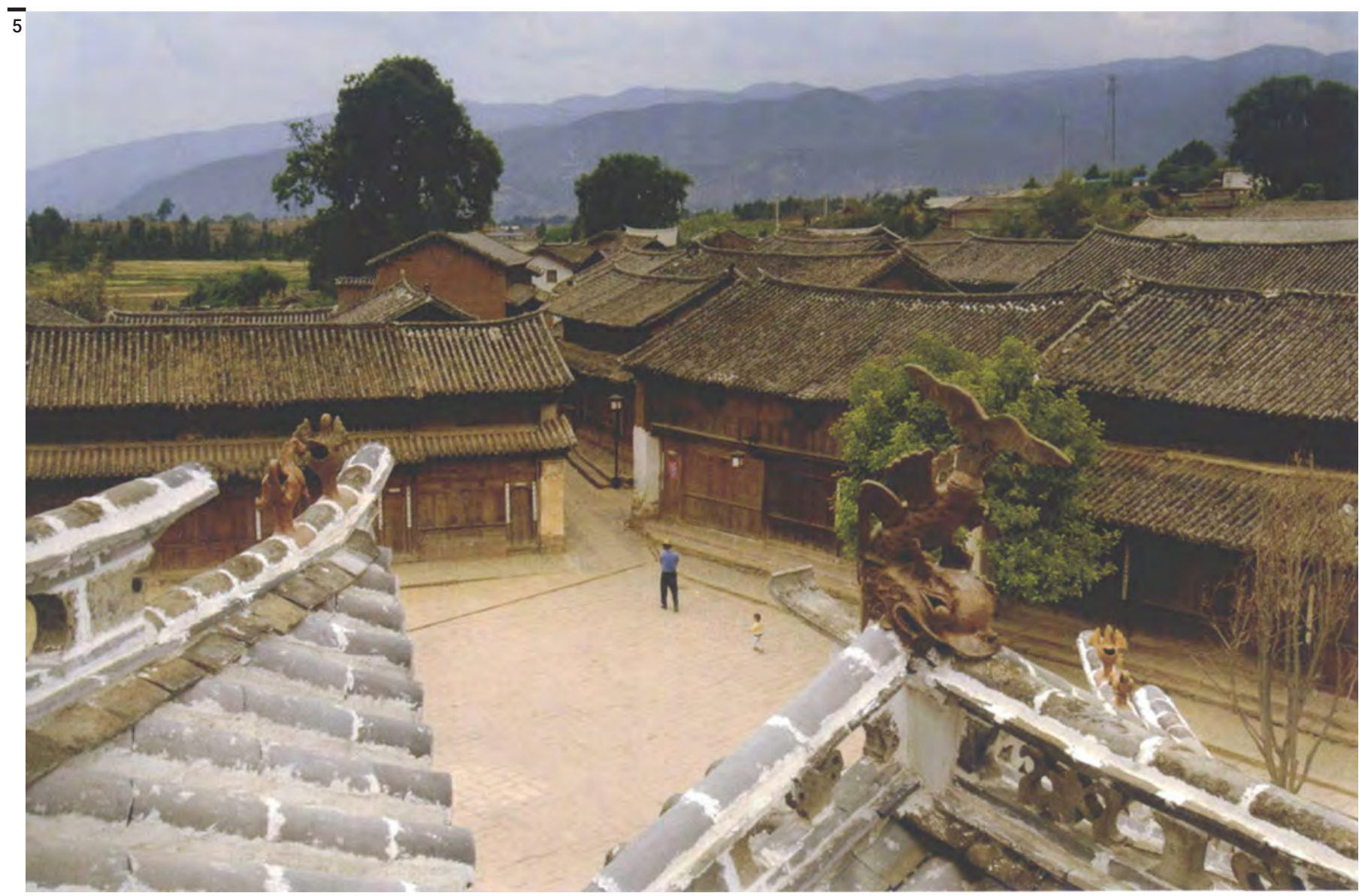




\section{Bibliografia}

Ahlers Anna (2014), Rural Policy Implementation in Contemporary China: New Socialist Countryside, Routledge. Ahlers Anna, Schubert Gunter (2009), «"Building a New Socialist Countryside" - Only a Political Slogan?», in Journal of Current Chinese Affairs, vol. 38, n. 4, pp. 35-62

Bolchover Joshua (2014), Rural urban framework: Transforming the Chinese countryside, Birkhäuser, BaselBoston-Berlin.

Bruno Edoardo, Tondo Dalila (2020), «"Senza mostrare i muscoli”: le librerie di Tao+C e Atelier AZL nel solco della "prosperosa società" della Cina contemporanea», in Archalp, nuova serie, n. 5, Politecnico di Torino-BUP, Torino-Bologna, pp. 87-93.

Lin John, Devabhaktuni Sony (2020), As found houses: Experiments from self-builders in rural China, Applied Research + Design Publishing, San Francisco.

Liu Wenling, Spaargaren Gert, Mol Arthur P.J., Heerink Nico, Wang Can (2014), «Low carbon rural housing provision in China: Participation and decision making», in Journal of Rural Studies, n. 35, pp. 80-90.

Renfer Christian (2017), «Considerations of a Swiss Monument Preservationist during a Visit to Traditional Villages in China: The Yunnan Shaxi Rehabilitation Project as an Opportunity», in Built Heritage, vol. 1, n. 1, pp. 57-66. Rozelle Scott, Hell Natalie (2020), Invisible China: How the Urban-Rural Divide Threatens China's Rise, Chicago, IL, University of Chicago Press.

Sigley Gary (2010), «Cultural Heritage Tourism and the Ancient Tea Horse Road of Southwest China», in International Journal of China Studies», vol. 2, n. 1, pp. 531-544

Fig. 6 Van der Ploeg Jan Douwe (2019), Agricoltura e contadini nella Cina d'oggi, Donzelli, Roma.

II Padiglione Kui Wong Edward (2016), «An Ancient Caravan Town in China Is Reborn», in The New York Times, March 27.

dopo l'intervento di

recupero

Yang Jinkung, Hui Eddie, Lang Wei, Li Xun (2018), «Land Ownership, Rent-Seeking, and Rural Gentrification: Reconstructing Villages for Sustainable Urbanization in China», in Sustainability, vol. 10, n. 6, pp. 1-17.

Fig. 7

Soluzione con giunzioni a tenone e mortasa come rinforzo strutturale per i manufatti di Shaxi.

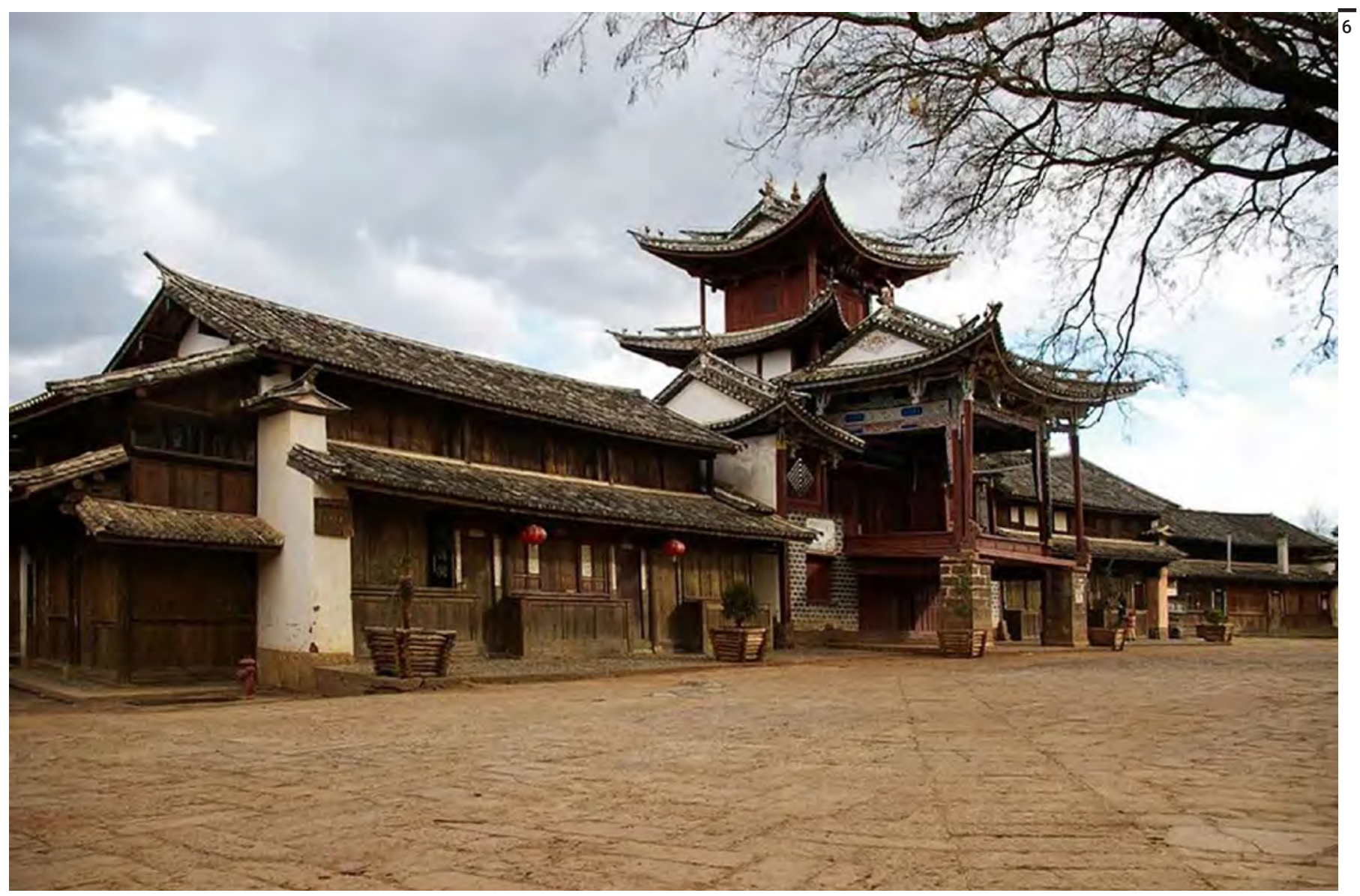




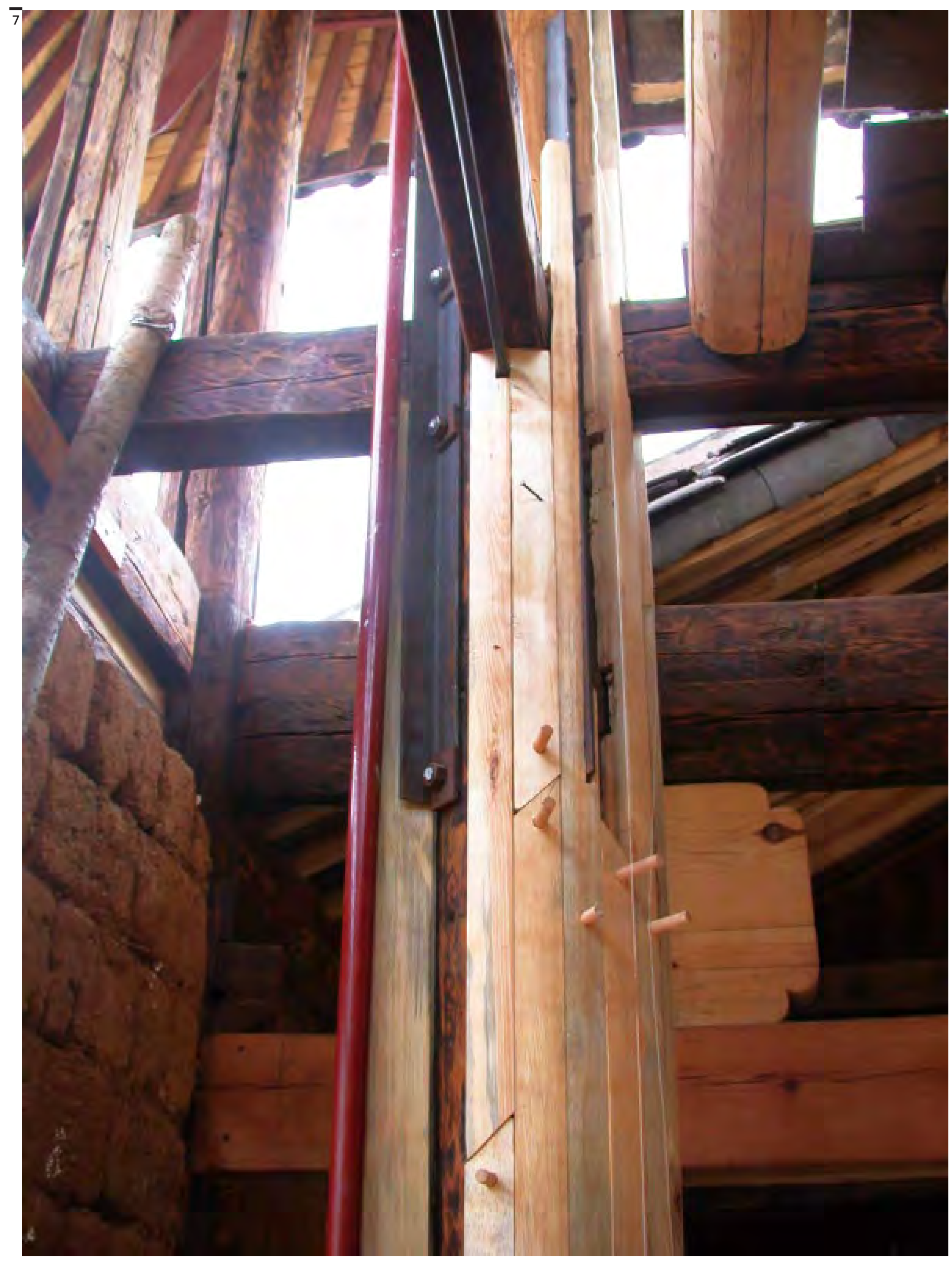

